\title{
Assessment of Thermophysical and Mechanical Properties of Composite Panels Fabricated from Untreated and Treated Coconut Husk Particles for Structural Application
}

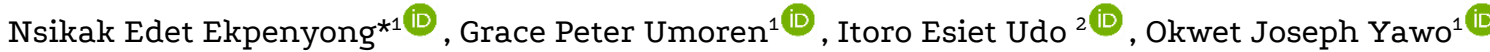 \\ ${ }^{1}$ Department of Physics, Akwa Ibom State University, Ikot Akpaden, Mkpat Enin, Akwa Ibom State, Nigeria \\ ${ }^{2}$ Deptartment of Chemistry, University of Uyo, Uyo, Akwa Ibom State, Nigeria
}

\section{Keywords}

Alkaline treatment

Bulk density,

Ceiling,

Flexural strength,

Nailability.

\begin{abstract}
Large quantities of coconut husks generated are under-utilized. In developing countries, this situation warrants their disposal by open burning or indiscriminate dumping. Either practice adversely affects the environment and public health. In this study, test samples were fabricated from untreated coconut husk particles (UCP) at $0 \%, 25 \%, 50 \%, 75 \%$, and $100 \%$ weight proportions with treated coconut husk particles (TCP) using epoxy resin as binder. Five representative samples were prepared per formulation and then subjected to various intended tests. The test results revealed water absorption, bulk density, thermal conductivity, specific heat capacity, thermal diffusivity, nailability, flexural strength, and compressive strength to be $(24.88 \pm 0.04) \%,(459.78 \pm 0.05) \mathrm{kgm}^{-3},(0.0867 \pm 0.0001) \mathrm{Wm}^{-1} \mathrm{~K}^{-1}$, (1573.76 \pm 1.14$) \mathrm{Jkg}^{-1} \mathrm{~K}^{-1},(1.198 \pm 0.002) \times 10^{-7} \mathrm{~m}^{2} \mathrm{~s}^{-1},(100.0 \pm 0.0) \%,(11.94 \pm 0.03) \mathrm{N} / \mathrm{mm}^{2}$, and $(22.86 \pm 0.04) \mathrm{N} / \mathrm{mm}^{2}$ respectively for the control sample with UCP content. Also, the respective values of the properties were $(29.05 \pm 0.06) \%,(583.87 \pm 0.05) \mathrm{kgm}^{-3},(0.1009 \pm$ $0.0002) \mathrm{Wm}^{-1} \mathrm{~K}^{-1},(1402.66 \pm 1.45) \mathrm{Jkg}^{-1} \mathrm{~K}^{-1},(1.232 \pm 0.004) \times 10^{-7} \mathrm{~m}^{2} \mathrm{~s}^{-1},(100.0 \pm 0.0) \%,(14.58 \pm$ $0.03) \mathrm{N} / \mathrm{mm}^{2}$, and $(33.27 \pm 0.02) \mathrm{N} / \mathrm{mm}^{2}$ for the counterpart sample containing the TCP. All the samples showed better tendencies for thermal insulation performance compared to conventional ceilings like plywood, PVC, and asbestos. Thus, utilization of coconut husks as described in this study could alternatively help to solve the disposal problems of such wastes while availing building industries with suitable raw materials to manufacture cost-effective heat-insulating ceilings.
\end{abstract}

\section{Introduction}

Coconut (Cocos nucifera Linnaeus) belongs to the palm family known as Arecaceae and it is available yearly. Essentially, coconut grows under varying climatic and soil conditions in the bountiful tropical coastal areas of Africa, Asia, Central America, and Oceania. When grown in sandy soil and annual rainfall is regular with high humidity, coconut tree can thrive very well and bear fruits yearly for about 70 years [1]. Globally, coconuts are planted on more than 10 million hectares in 92 countries [2]. According to Burton [3], Indonesia alone produces $18,300,000$ tons of coconuts. Coconut is well-known for its versatility and as such, it is referred to as the"tree of heaven". Traditionally, it is often used as food and in cosmetic products $[4,5]$ In addition to energy, coconut plantations serve as a source of various useful products [2].

Among the various residues that could be generated from harvesting/processing of coconuts, the husks and shells appear to be prominent. Utilization of these residues (coconut shells and husks) has been reported in few studies and it has been revealed that they have potential uses in design/development of certain building materials. For instance, coconut husk can be used to improve the compressive and flexural strength of concrete [6]. Agham [7] noticed reduction in thermal conductivity of polyurethane foam when mixed with coconut husk for use as an insulating material. In their studies, Robert et al [8] found that partial replacement of sand with $20 \%$ to $30 \%$ of coconut husk could yield solid core sandcrete blocks of satisfactory performance for non-load bearing applications. Enhancement of concrete durability using coconut shell and fiber [9] and fabrication of composite ceiling panels from coconut husks, waste newspaper and cassava starch [10] have been reported as well. According to Halfhide et al [11], spent coconut husks can be utilized for production of useful sorbent for small decentralization of waste water treatment systems.
In line with the findings that a whole coconut fruit can yield 14 to $15 \%$ shell and 47 to $50 \%$ husk as wastes [12], it has been observed that coconut husk is generated in large quantities but highly underutilized. Due to ineffective solid waste management system in Nigeria and other developing countries [13,14], a vast amount of coconut husk is either openly burnt or indiscriminately dumped as a prevalent disposal technique for the waste. Such practices pose a serious threat to the environment and public health. It has been noticed that one of the ways of ensuring inexpensive building construction is by tailoring solid wastes into development of value-added building materials [15] Considering the fact that housing is a basic human need and thermal comfort is a necessity in buildings, this work is aimed at utilizing coconut husks with epoxy resin in development of thermal insulation panels. Thermophysical and mechanical properties of control and composite panels developed from untreated and treated coconut husk particles will be assessed to ascertain their suitability for structural application. While the knowledge of thermal properties is vital in the choice of a material for construction of self-cooled buildings [16], mechanical properties reflect the relationship between the material's response to/or deformation from an applied load. This undertaking, being the first to be reported, is believed to be of great benefits to researchers, engineers, builders, and building material scientists.

\section{Experimental Work}

\subsection{Materials}

Coconut husks (discarded as waste), cellophane, sodium hydroxide pellets, epoxy resin (K7001) with its hardener, and tap water were used as the major materials in this work. The husks were collected from local coconut growers whereas the epoxy resin was bought from building materials shop. These materials were obtained in large quantities within Uyo Local Government Area, Akwa Ibom State, Nigeria. 


\subsection{Processing and analysis of the coconut husks}

The husks were washed with water in order to remove accompanying dirts from them before they were cut into smaller pieces and then sun-dried completely in air. The dried pieces of the husks were blended using a household electric blender and the particles obtained were sieved. The quantity of it that passed through size 10 Mesh of US sieve was divided into two halves. One half of it was chemically treated using a freshly-prepared alkaline solution containing $100 \mathrm{~g}$ of sodium hydroxide pellets in $1000 \mathrm{~cm}^{3}$. This treatment lasted for 6 hours in a plastic bucket after which the particles were removed, thoroughly washed, and then subjected to complete sun-drying. In order to determine the chemical composition of the processed husks, a required quantity (in each case of the untreated and treated particles) was analysed using gravimetric method as employed by Ayeni et al [17]. This was done for five times. Then after, the mean and corresponding standard error values of the results were determined.

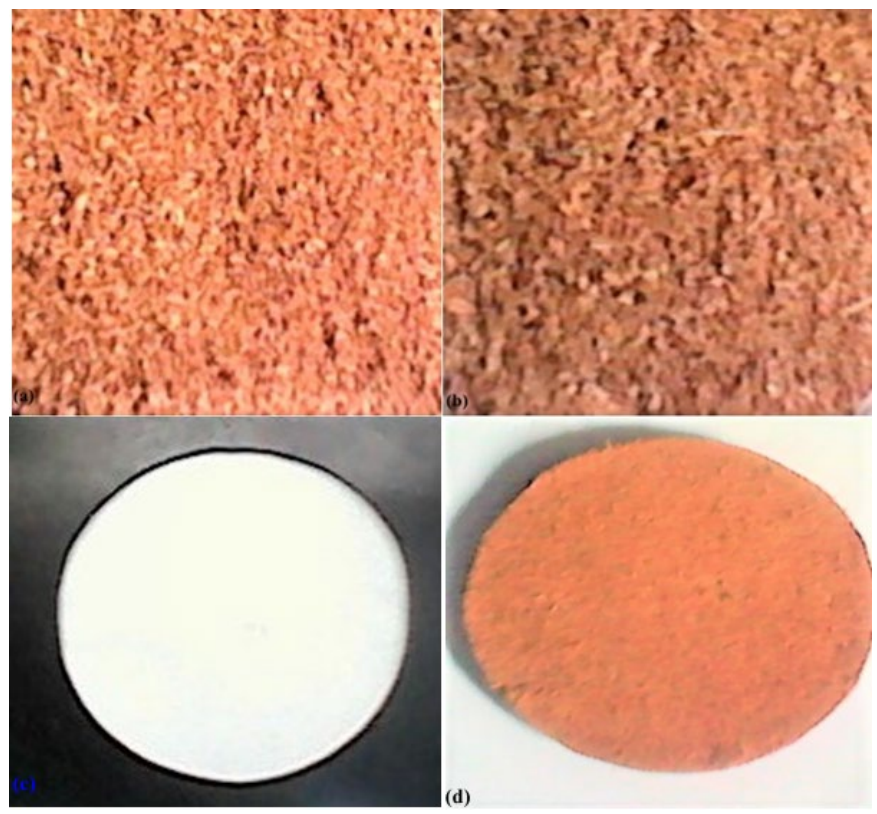

\subsection{Preparation of test samples}

Samples in this study were fabricated by hand lay-up technique. The untreated coconut husk particles were mixed with binder in a 1:2 weight ratio (of the husk to binder). The binder used was prepared by mixing equal weight proportions of the epoxy resin and its hardener. A thorough mixture of the husk particles with binder was cast into a circular mold measuring $110 \mathrm{~mm}$ in diameter and $9 \mathrm{~mm}$ in thickness (based on the requirements for the thermal conductivity measurement method to be applied in the study). Before casting, the inner surface of the mold was covered with a clean cellophane material so as to ease removal of the sample. Compaction was done for 1 hour by means of a laboratory-made compacting machine maintained at $5 \mathrm{kN}$. On removal, the sample was cured for 2 days under ambient conditions. After that, it was subjected to continuous sun-drying and weighing until no further reduction in its weight was observed. Other samples, including those made by utilizing the treated coconut husk particles at $25 \%, 50 \%, 75 \%$, and $100 \%$ weight proportions were similarly prepared. In each case, five representative test samples were developed. The samples prepared were subjected to various property tests. Figure 1 shows the pictures of the untreated and treated coconut husk particles, mold, and one of the developed samples.

\subsection{Property tests}

\subsubsection{Thermophysical properties}

Thermal conductivity was evaluated for each sample using Modified Lee -Charlton's Disc Apparatus Technique described in details elsewhere [18]. Heating was done by means of an electric hotplate (Lloytron E4102WH) and the rate of cooling modeling was performed with the aid of Origin Software Version 2019. The data obtained were used to compute the required thermal conductivity, $k$ as

$k=\left(\frac{M c x}{A \Delta \theta}\right) \frac{d T}{d t}$

where $M=$ mass of the disc, $c=$ specific heat capacity of the disc, $x=$ sample's thickness, $A=$ cross-sectional area of the sample, $\Delta \theta=$ temperature across the sample's thickness and $\frac{d T}{d t}=$ rate of cooling of the disc.

Each of the samples was then cut into shapes and sizes suitable for other intended tests in this work.

For each sample, the mass, $M_{s}$ was measured by means of a digital balance (S. METTLER- 600g) and the volume, V was determined by Modified Water Displacement Method [19] before the corresponding bulk density, $\rho$ was calculated thus,

$\rho=\frac{M_{S}}{V}$

For water absorption determination, immersion method was used. The mass $M_{d}$ of each sample before immersion was measured. The immersion of the samples lasted for 24 hours before the samples were removed and allowed to surface-dry. The mass $M_{w}$ after immersion was also determined and water absorption was found based on the relation [10]

$W A=\left(\frac{M_{w}-M_{d}}{M_{d}}\right) 100 \%$

where $W A=$ water absorption of the sample.

With the aid of SEUR'S Apparatus [20], the specific heat capacity, $c$ was measured for each of the samples. In addition to the test sample, the other two accessories used for heat exchange were aluminium plate and plywood plate, each measuring $50 \mathrm{~mm} \times 50 \mathrm{~mm} \times 10 \mathrm{~mm}$. The values of thermal conductivity, bulk density, and specific heat capacity obtained for each sample were applied for calculation of the corresponding thermal diffusivity, $\lambda$ according to the formula [10]

$\lambda=\frac{k}{\rho c}$

\subsubsection{Mechanical properties}

For evaluation of nailability, a nail of length $50 \mathrm{~mm}$ was used. The nail was driven by means of a carpenter's hammer into each sample through the thickness. When either a visible crack or successful nail penetration was observed, the process was discontinued. In either case, the depth, $d$ of nail penetration and thickness, $x$ were used to determine nailability as [21]

$N_{b}=\left(\frac{d}{x}\right) 100 \%$

The flexural strength was determined by three-point bending method [22] using a Universal Testing Machine (WDW - 10). In this case, the test sample measuring $80 \mathrm{~mm}$ x $15 \mathrm{~mm}$ x $9 \mathrm{~mm}$ was suspended as a single beam supported at two points on the flexure of the machine. The test speed applied was $5.0 \mathrm{~mm} \mathrm{~min}^{-1}$ and the data obtained when the sample fractured were employed to compute the flexural strength, $f_{s}$ according to the relation

$f_{s}=\frac{3}{2}\left(\frac{F_{\max } L}{b x^{2}}\right)$

where $L=$ length of span of the sample, $F_{\max }=$ load recorded at fracture moment, $b$ = width of the sample, $x=$ sample's thickness.

For compressive strength evaluation, the test protocols outlined in [23] were followed and a cross-head speed of $5 \mathrm{~mm} / \mathrm{min}$ was applied. When the sample failed to survive the stress on it, the maximum force, $F$ at that instant was used with the sample's cross-sectional area, $A$ to calculate the required compressive strength, $C S$ thus $C S=\frac{F}{A}$

All the tests were conducted at room temperature with $\pm 1.0^{\circ} \mathrm{C}$ variations and the mean values for each formulation were computed with corresponding standard erro values. 


\section{Results and Discussion}

Table 1 shows the proportion of each lignocellulosic constituent in the untreated as well as treated coconut husk particles utilized in this study. In table 2 , the results of properties investigation performed on the fabricated samples are recorded. The samples that contain $100 \%$ of either the UCP or TCP are control while others are regarded as composite samples in this work.

Table 1. Chemical compositions of the coconut husk particles

\begin{tabular}{lcc}
\hline Lignocellulosic & \multicolumn{2}{c}{ Proportions (\%) } \\
\cline { 2 - 3 } constituents & UCP & TCP \\
\hline Cellulose & $24.26 \pm 1.02$ & $34.08 \pm 0.47$ \\
Hemicelluloses & $20.54 \pm 1.31$ & $17.28 \pm 0.29$ \\
Lignin & $43.81 \pm 1.22$ & $35.14 \pm 0.52$ \\
\hline \multicolumn{2}{c}{ UCP $=$ Untreated Coconut husk Particles } \\
\multicolumn{2}{c}{$T C P=$ Treated Coconut husk Particles }
\end{tabular}

It can be seen in Table 1 that the TCP contains greater fraction of cellulose but less proportions of hemicelluloses and lignin compared to the UCP. On average, the alkaline treatment leads to removal of up to $3.26 \%$ and $8.67 \%$ contents of hemicelluloses and lignin respectively. However, it increases the cellulose fraction by a difference of $9.82 \%$. Keeping in mind that cellulose is highly hydrophilic whereas lignin is highly hydrophobic, this observation portrays that UCP and TCP can exhibit different behavioural tendencies.

Table 2. Results of property tests performed on the fabricated samples

\begin{tabular}{|c|c|c|c|c|c|c|c|c|}
\hline $\begin{array}{c}\text { Mix } \\
\text { propor } \\
\text { tions } \\
(\%) \\
\text { UCP:T } \\
\text { CP }\end{array}$ & $\begin{array}{c}\text { Water } \\
\text { absorp } \\
\text { tion, } \\
W A \\
(\%)\end{array}$ & $\begin{array}{c}\text { Bulk } \\
\text { density, } \\
\rho \\
\left(k^{-3} m^{-3}\right)\end{array}$ & $\begin{array}{c}\text { Thermal } \\
\text { conductivity, } \\
k \\
\left(W m^{-1} K^{-1}\right)\end{array}$ & $\begin{array}{c}\text { Specific } \\
\text { heat } \\
\text { capacity, } c \\
\left(\mathrm{Jg}^{-1} \mathrm{~K}^{-1}\right)\end{array}$ & $\begin{array}{c}\text { Thermal } \\
\text { diffusivity, } \\
\lambda \\
\left(10^{-7} \mathrm{~m}^{2} \mathrm{~s}^{-1}\right)\end{array}$ & $\begin{array}{c}\text { Nailability, } \\
N_{b} \\
(\%)\end{array}$ & $\begin{array}{c}\text { Flexural } \\
\text { strength, } \\
f_{s} \\
\left(\mathrm{~N} / \mathrm{mm}^{2}\right)\end{array}$ & $\begin{array}{c}\text { Compressive } \\
\text { strength, } \\
C S \\
\left(\mathrm{~N} / \mathrm{mm}^{2}\right)\end{array}$ \\
\hline \multirow[t]{2}{*}{$0: 100$} & 29.05 & 583.87 & 0.1009 & 1402.66 & 1.232 & 100.0 & 14.58 & 33.27 \\
\hline & \pm 0.06 & \pm 0.05 & \pm 0.0002 & \pm 1.45 & \pm 0.004 & \pm 0.0 & \pm 0.03 & \pm 0.02 \\
\hline \multirow[t]{2}{*}{$25: 75$} & 27.68 & 552.46 & 0.0979 & 1450.03 & 1.222 & 100.0 & 13.41 & 28.91 \\
\hline & \pm 0.02 & \pm 0.07 & \pm 0.0001 & \pm 1.41 & \pm 0.002 & \pm 0.0 & \pm 0.03 & \pm 0.02 \\
\hline \multirow[t]{2}{*}{$50: 50$} & 26.24 & 520.79 & 0.0948 & 1498.73 & 1.215 & 100.0 & 12.82 & 25.63 \\
\hline & \pm 0.05 & \pm 0.06 & \pm 0.0002 & \pm 1.37 & \pm 0.003 & \pm 0.0 & \pm 0.01 & \pm 0.03 \\
\hline \multirow[t]{2}{*}{$75: 25$} & 25.02 & 495.88 & 0.0921 & 1539.89 & 1.206 & 100.0 & 12.25 & 24.29 \\
\hline & \pm 0.02 & \pm 0.03 & \pm 0.0002 & \pm 1.20 & \pm 0.002 & \pm 0.0 & \pm 0.02 & \pm 0.03 \\
\hline \multirow[t]{2}{*}{ 100:0 } & 24.88 & 459.78 & 0.0867 & 1573.76 & 1.198 & 100.0 & 11.94 & 22.86 \\
\hline & \pm 0.04 & \pm 0.05 & \pm 0.0001 & \pm 1.14 & \pm 0.002 & \pm 0.0 & \pm 0.03 & \pm 0.04 \\
\hline
\end{tabular}

In Table 2, the value of water absorption of sample containing $0 \%$ of the TCP is $(4.17 \pm 0.07) \%$ greater than the value obtained for the one similarly prepared but with the UCP. This is possible because cellulose proportion is greater in the TCP than in the case of the UCP. Consequently, the water- absorbing ability of the developed samples increases with increasing proportions of the TCP. Because of the binder used, the maximum water absorption percentage is lower for the samples compared to the value of $90.12 \%$ reported by Etuk et al [24] for binderless sugarcane leaf board and the value of $86.33 \%$ reported by Robert et al [1] for coconut leaflet board, both of which are recommended for use as ceiling in building design.

At $100 \%$ level of utilization, the sample with UCP content only is less dense than the one fabricated using the TCP. Considering the fact that during fabrication the same total volume and other factors were maintained, it means that application of alkaline treatment to obtain the TCP brings about cell wall densification. This makes the TCP to become heavier than the UCP. From the results presented, the bulk density values of the control samples yield a difference of $(124.09 \pm$ $0.07) \mathrm{kgm}^{-3}$. By increasing the proportion of the TCP from $0 \%$ to $25 \%$, $50 \%$, and $75 \%$ in the samples, the mean bulk density values obtained increase by a difference of $36.10,61.01$, and 92.68 (all in $\mathrm{kgm}^{-3}$ ) respectively. This simply implies that, in terms of absorption of dead loads in buildings, the sample containing 100\% UCP can perform better than the one fabricated with the TCP at any content level. However, all the samples may be regarded as low-density panels based on the standard criteria stipulated in [25].

The obtained value of thermal conductivity at 0\% TCP level is (16.28 \pm $0.23) \%$ lower than the value obtained for the sample made with the UCP at similar level. This is plausibly due to the fact that untreated coconut husk is made up of more light dusty particles than the alkalitreated one. As such, a greater volume of air occupies the voids in the sample containing the UCP compared to the case of sample made with the TCP. This fact is supported by the findings of Robert et al [15] in the cases involving the use of untreated and treated wood dust with plaster of Paris (POP). Thus, since air is one of the best thermal insulators, transmission of heat can be restricted more with the use of the UCP compared to utilization of the TCP. As can be deduced from the results obtained, thermal resistance (ratio of thickness to thermal conductivity) values are about $0.104 \mathrm{~W}^{-1} \mathrm{~m}^{2} \mathrm{~K}$ and $0.089 \mathrm{~W}^{-1} \mathrm{~m}^{2} \mathrm{~K}$ for the control samples developed using the UCP and TCP respectively. This substantiates the above assertion that the use of UCP ensures better thermal insulation efficiency than making use of the TCP. For samples containing the UCP and TCP, the largest difference in mean thermal conductivity values (as a result of varying the proportion of the TCP) is $0.0054 \mathrm{Wm}^{-1} \mathrm{~K}^{-1}$ and is possible only when $75 \%$ of the UCP and $25 \%$ of the TCP are utilized in the composite mix. This therefore suggests that the best thermal insulation performance could be achieved from composite samples developed using the UCP and TCP at such content levels. That notwithstanding, the thermal conductivity values obtained for the samples are within the range given by Rajput [26] as $0.023 \mathrm{Wm}^{-1} \mathrm{~K}^{-1}$ to $2.900 \mathrm{Wm}^{-1} \mathrm{~K}^{-1}$ for heat-insulating and construction materials. Figure 2 shows that bulk density and thermal conductivity trend positively with increasing proportions of the TCP. This indicates decrease in lightness and enhancement of heat transmission as the fraction of the TCP increases. A sharp variation is noticed when the content of TCP is greater than $25 \%$ in either case.

Additionally, based on the specific heat capacity and thermal diffusivity values obtained for the samples, it could be averred that utilization of the UCP at $75 \%$ level with TCP at $25 \%$ level is optimum for fabrication of composite samples capable of exhibiting the best thermophysical and mechanical performance in buildings. For instance, at the said levels of UCP and TCP composition in the sample, about $1539.89 \mathrm{~J}$ of heat is required to change the temperature of a unit mass of the sample by one Kelvin. Also, the mean specific heat capacity value is $33.87 \mathrm{Jkg}-{ }^{1} \mathrm{~K}^{-1}$ less than the value obtained for the control sample made using the UCP. This difference is the least decrement among others that result from increasing the TCP content in steps of $25 \%$. Again, for other cases, the increase in thermal conductivity values resulting from increment in the proportion of the TCP indicates decrease in thermal insulation efficiency.

Furthermore, since thermal conductivity and bulk density resonate with each other in line with the empirical relationship expressed as eq. 4 in this work, it can be deciphered that high specific heat capacity and low thermal conductivity reduce thermal diffusivity of the studied samples. From the obtained results, the mean thermal diffusivity values of the composite samples increase by $0.67 \%, 1.42 \%$, 
and $2.00 \%$ due to utilization of the TCP at $25 \%, 50 \%$, and $75 \%$ levels respectively. These increments in thermal diffusivity are feasible because the use of TCP alone yields sample that has less heat-storing ability with greater heat diffusion tendency than its counterpart made using the UCP. By implication, if both control samples are subjected to thermal front under same conditions in a building, heat spread with temperature propagation will be faster in the case of applying sample made using the TCP compared to application of the one similarly developed using the UCP.

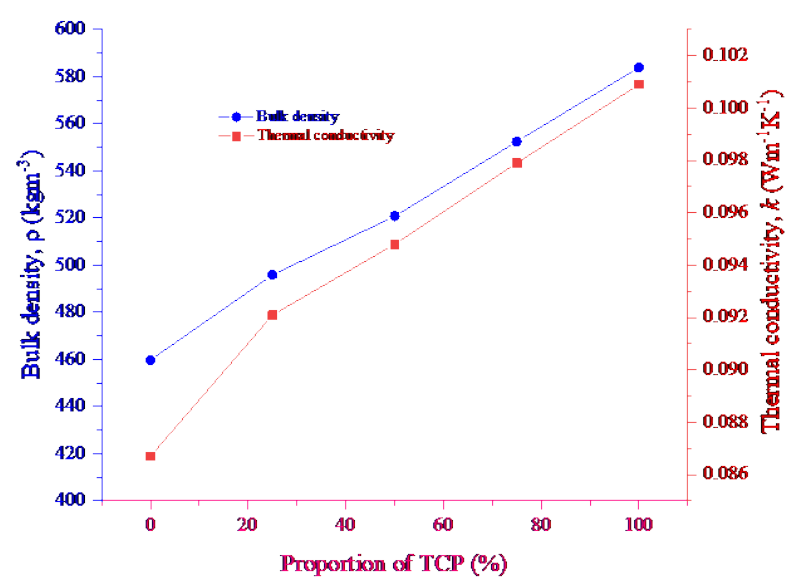

Figure 2. Variations of bulk density and thermal conductivity with TCP content

Though the results reveal nailability of $100 \%$ for all the samples thus indicating that they can withstand nailing, the values of flexural strength vary slightly. With $100 \%$ content of the UCP, the flexural strength of the sample produced is $(22.11 \pm 0.34) \%$ less than the value obtained for the sample developed at a similar level using the TCP. This signifies that internal bond strength is greater with the use of the TCP than when UCP is utilized. Meanwhile, the morphology of the raw fiber (that is, UCP) is changed by the alkaline treatment, resulting in the TCP having rougher surface than the UCP. Thus, a greater adhesion is ensured at the interface between the binder and the TCP unlike in the case of using the UCP. Since the ability to withstand bending stress is directly proportional to the existing internal bond strength, it is obvious that sample made with the TCP alone has a greater ability than the control sample containing the UCP to resist any stress tending to rupture them. Consequently, flexural strength increases with the proportion of the TCP in the composite samples. This same reason accounts for the compressive strength, showing that the fabricated panels can withstand a reasonable amount of stress before they shatter. The high mechanical strengths exhibited by the samples signify that they are suitable panels for partition purpose in buildings.

Table 3 shows the characterization of some common conventional heat-insulating ceiling materials as reported in the literature. Comparatively, the studied samples have bulk density values that are far less than those of PVC and asbestos. The maximum bulk density value obtained for the samples in this work compares well with the value of $589.0 \mathrm{kgm}^{-3}$ reported for plywood and the maximum bulk density value obtained shows a good comparison with the value of $480.0 \mathrm{kgm}^{-3}$ reported for laminated paperboard. In terms of thermal conductivity, each of the materials like plywood, PVC, and asbestos is observed to have a higher value whereas the fibreboard and paperboard have lower values than the studied samples. However, the specific heat capacity value reported for the fibreboard is $40.27 \%$ lower than the mean minimum value $\left(1402.66 \mathrm{kgm}^{-3}\right)$ obtained for the samples in this work. Also, sample containing $100 \%$ of the UCP has greater specific heat capacity than paperboard though they compare well in terms of density. As a matter of fact, the samples possess properties that could enable them perform satisfactorily if used as ceiling panels in building design. Their low density makes them have choice preference over PVC, asbestos, and plywood as far as absorption of dead loads and retardation of heat are considered as priorities. When considering storage of heat to prolong temperature fluctuation, the studied samples could perform better than PVC, asbestos, fibreboard, and paperboard.

Table 3. Characterization of some conventional ceiling panels as reported in the literature

\begin{tabular}{lccrc}
\hline Material & $\begin{array}{c}\text { Density } \\
\left(\mathrm{kgm}^{-3}\right)\end{array}$ & $\begin{array}{c}\text { Thermal } \\
\text { conductivity } \\
\left(\mathrm{Wm}^{-1} \mathrm{~K}^{-1}\right)\end{array}$ & $\begin{array}{c}\text { Specific } \\
\text { heat } \\
\text { capacity } \\
\left(\mathrm{Jkg}^{-1} \mathrm{~K}^{-1}\right)\end{array}$ & Source \\
\hline Plywood & 589.0 & 0.177 & 2500.0 & {$[27]$} \\
PVC & 1379.0 & 0.160 & 1004.0 & {$[28]$} \\
Asbestos & 1810.0 & 0.370 & 848.0 & {$[29]$} \\
Fibreboard & 300.0 & 0.060 & 1000.0 & {$[28]$} \\
Paperboard & 480.0 & 0.072 & 1400.0 & {$[28]$} \\
(Laminated) & & & & \\
\hline
\end{tabular}

\section{Conclusion}

Experimental results from this study revealed that control sample fabricated using untreated coconut husk particles (UCP) has better thermophysical properties than its counterpart made from treated coconut husk particles (TCP). Nailability values were found to be $100 \%$ in all the cases. However, the control sample containing TCP possessed flexural strength and compressive strength that are about $22.11 \%$ and $33.83 \%$, respectively, greater than the values obtained for the panel similarly developed using UCP. On the contrary, the use of UCP at $100 \%$ level by weight yielded samples with improved thermal insulation tendencies over their counterparts by decreasing thermal conductivity, specific heat capacity, and thermal diffusivity by $14.07 \%, 12.20 \%$, and $2.76 \%$ respectively. It was also observed that water absorption and bulk density of the composite samples increased with increasing proportions of the TCP. On comparing with conventional ceiling panels, it was found that the samples could exhibit more satisfactory and better thermal insulation performance than plywood, PVC, and asbestos commonly applied in buildings. Hence, utilization of wastes like coconut husks for production of ceiling panels is, no doubt, a promising alternative for ensuring availability of low-cost thermal-insulating ceilings for building purposes. In turn, the disposal problems associated with the wastes in question (coconut husks) could be solved in order to minimise their resultant adverse effects on environment and public health while improving the economy.

\section{Declaration of Conflict of Interests}

The authors declare that there is no conflict of interest. They have no known competing financial interests or personal relationships that could have appeared to influence the work reported in this paper.

\section{References}

[1.] Robert, U.W., Etuk, S.E., Agbasi, O.E., Umoren, G.P., Inyang, N.J., Investigation of thermophysical and mechanical properties of board produced from coconut (Cocos nucifera) leaflet, Environmental Technology \&Innovation, 24(1) (2021) 101869 https://doi.org/10.1016/j.eti.2021/101869.

[2.] Zafar, S, Energy Potential of Coconut Biomass, Bioenergy Consult, (2021) https://www.bioenergyconsult.com/coconutbiomass/ [Accessed March 19, 2021].

[3.] J. Burton, J., The World LeadersIn Coconut Production, (2019) Available:

https://www.worldatlas.com/articles/the-world-leaders-incoconut-production.html

[4.] Madakson, P.B., Yawas, D.S., Apasi, A., Characterization of coconut shell ash for potential utilization in metal matrix composites for automotive applications, Intl J Eng Sci Techn. 4 (2012) $1190-1198$.

[5.] Tan, I.A.W., Ahmad, A.I., Hameed, B.H., Preparation of activated carbon from coconut husk: study on removal of $2,4,6-$ trichlorophenol using response surface methodology, Journal of Hazard Materials, 153 (2008) 709 - 717 
[6.] Ede, A.N., Agbede, J.O., Use of coconut husk for improved compressive and flexural strength of concrete, Interrnational Journal of Science and Engineering Research, 6(2) (2015) 968 974

[7.] Agham, R.D., Performance of coconut husk mixed polyurethane foam as an alternative insulating material, International Journal of Engeering, Innovation and Technology, 2(9) (2013) 100 - 104.

[8.] Robert, U.W., Etuk, S.E., Agbasi, O.E., Ekong, S.A., Properties of Sandcrete Block produced with Coconut Husk as Partial Replacement of Sand, Journal of Building Materials and Structures, 7 (2020) 95 - 104

[9.] Sekar, A., Kandasamy, G., Study on Durability Properties of Coconut Shell Concrete with Coconut Fiber, Buildings, 9(5) (2019) 107.

[10.] Robert, U.W., Etuk, S.E., Umoren, G.P., Agbasi, O.E., Assessment of thermal and mechanical properties of composite board produced from coconut (Cocos nucifera) husks, waste newspapers, and cassava starch, International Journal of Thermophys, 40 (2019) 83.

[11.] Halfhide, T., Lalgee, L.J., Singh, K.S., Williams, J., Sealy, M., Manoo, A., Mohammed, A., Nutrient removal using spent coconut husks, $\mathrm{H}_{2}$ Open Journal, 2(1) (2019) 125 - 136

[12.] Obeng, G.Y., Amoah, D.Y., Opoku, R., Sekyere, C.K.K., Adjei, E.A., Mensah, E., Coconut Wastes as Bioresource for Sustainable Energy: Quantifying Wastes, Calorific Values and Emissions in Ghana, Energies, 13 (2020) 2178.

[13.] Babayemi, J.O., Dauda, K.T., Evaluation of solid wastes generation, categories, and disposal options in developing countries: A case study of Nigeria, Journal of Applied Science and Environmental Management, 13(3) (2009) $83-88$.

[14.] Elemile, O.O., Sridhar, M.K.C., Oluwatuyi, O.E., Solid waste characterization and its recycling potential: Akure municipal dumpsite, South Western Nigeria, Journal of Material Cycle and Waste Management, 21(3) (2019) 583 - 593.

[15.] Robert, U.W., Etuk, S.E., Agbasi, O.E., Ekong, S.A., Abdulrazzaq, Z.T., Anonaba, A.U., Investigation of Thermal and Strength Properties of Composite Panels fabricated with Plaster of Paris for Insulation in Buildings, International Journal of Thermophysics., 42(2) (2021) 25.

[16.] Akpabio, L.E., Ekpe, S.D., Etuk, S.E., Essien, K.E., Thermal properties of Oil and Raffia Palm Fibres, Global Journal of Pure and Applied Sci., 7(3) (2001) 575 - 578

[17.] Ayeni, A.O., Adeeyo, O.A., Oresegun, O.M., Oladimeji, T.E. Compositional analysis of lignocellulosic materials: Evaluation of an economically viable method suitable for woody and non-woody biomass, American Journal of Engineering Research, 4(4) (2015) 14 - 19.

[18.] Robert, U.W., Etuk, S.E., Agbasi, O.E., Okorie, U.S., Quick determination of thermal conductivity of thermal insulators using a Modified Lee - Charlton's Disc Apparatus Technique, International Journal of Thermophysics 42 (2021) 113.

[19.] Robert, U.W., Etuk, S.E., Agbasi, O.E., Modified Water Displacement Method and its Use for Determination of Bulk Density of Porous Materials, Journal of Renewable Energy and Mechnics, 1(1) (2019) 1 - 16.

[20.] Etuk, S.E., Robert, U.W., Agbasi, O.E., Design and Performance evaluation of a device for determination of specific heat capacity of thermal insulators, Beni-Suef University Journal of Basic and Applied Science, 9 (2020) 34.

[21.] Okorie, U.S., Robert, U.W., Iboh, U.A., Umoren Assessment of the suitability of tiger nut fibre for structural applications, Journal of Renewable Energy and Mechnics, 3(1) (2020) 32 - 39.
[22.] ASTM D790, Standard Test Methods for Flexural Properties of Unreinforced and Reinforced Plastics and Electrical Insulating Materials, ASTM International, West Conshohocken, PA. (2017)

[23.] ASTM C165, Standard Test Method for Measuring Compressive Properties of Thermal Insulations, International, West Conshohocken, PA. (2017).

[24.] Etuk, S.E., Agbasi, O.E., Robert, U.W., Investigation of heat transfer and mechanical properties of Saccharum officinarumleaf board, International Journal Energy Water Resources, (2021).

[25.] EN 312, particleboards specifications, European committee for Standardization, Brussels, Belgium. (2010).

[26.] Rajput, E.R.K., Heat and Mass Transfer, $6^{\text {th }}$ Revised edn., S. Chand and Company PVT Ltd, Ram Nagar, New Delhi, (2015) 15.

[27.] Gesa, F.N., Atser, A.R., Aondoakaa, I.S., Investigation of the thermal insulation properties of selected ceiling materials used in Markurdi metropolis (Benue State - Nigeria). American Journal of Engineering Research, 3(11) (2014) 245 - 250

[28.] Page, J., Lebens, R., Transmission properties of windows. Pilkington, (2018).

[29.] Oyekunle, J.A.O., Dirisu, J.O., Okokpujie, I.P., Asere, A.A. Determination of heat transfer properties of various PVC and non-PVC ceiling materials available in Nigerian markets, International Journal of Mechecanical Engineering Technology, 9(8) (2018) $963-973$

\section{How to Cite This Article}

Ekpenyong, N.E., Umoren, G.P., Udo, I.E., and Yawo, O.J., Assessment of Thermophysical and Mechanical Properties of Composite Panels Fabricated from Untreated and Treated Coconut Husk Particles for Structural Application, Brilliant Engineering, 2 (2022), 1-5. https://doi.org/10.36937/ben.2022.4547 